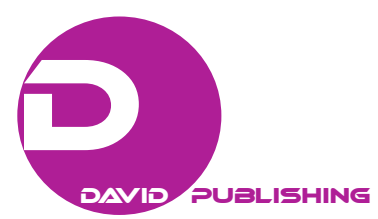

\title{
Factors Influencing the Choice of Social Network Sites in the Light of Cross-cultural Research
}

\author{
Małgorzata Bartosik-Purgat, Monika Guzek \\ Poznań University of Economics and Business, Poznań, Poland
}

\begin{abstract}
The main purpose of the paper is to identify the factors of social networking sites (SNS) that influence individual users' choice in foreign markets (cultures) and to determine endogenous characteristics (age and gender) differentiating this choice. Besides, the specific objective of the paper is to indicate interdependencies between the importance of particular factors of SNS and the devices used by users. The literature studies have been enriched with empirical studies carried out in China, Poland, and the United States. Empirical research was conducted with the use of a quantitative research method such as paper and electronic inquiry forms (CAWI), filled out by the respondents themselves. Referring to the importance of the characteristic features of SNS, it needs to be emphasized that the results of the empirical studies showed little differences among the researched countries. It was noted that the endogenous characteristics of users, such as gender and age, differentiate the significance of particular elements. The greatest interdependencies between the endogenous factors and the elements of SNS were observed in the Chinese group. The research also showed great significance of mobile devices in using SNS. The presented results have a significant application value for business entities creating SNS and their mobile applications in the foreign market .
\end{abstract}

Keywords: social networking sites, choice determinants, endogenous characteristics, foreign markets

\section{Introduction}

Nowadays, the Internet is a place where people perform many activities which so far have been known only in the offline world. The increased frequency of using the Internet raises the popularity of small sized smartphones, iPads, or tablets. Thanks to them, users can be constantly online. Greater frequency of using the Internet is caused by the appearance of SNS, which has changed and is still changing the activity of users on the Internet. Many of them cannot imagine living only offline life as SNSs enable cooperation, and allow users to actively participate in bilateral communication and involve in virtual life. Through SNS, users can, on the one hand, express their opinions on the products they have bought; while on the other hand, they actively participate in the process of creating new products, thus becoming prosumers. In fact, SNSs are a common platform for exchanging content created by users, they support active users and ensure the control of the content (e.g. inappropriate photos, posts offending social, ethnic groups, etc.) (Kaplan \& Haenlein, 2010).

\footnotetext{
Małgorzata Bartosik-Purgat, associate professor, Ph.D., Department of International Management, Poznań University of Economics and Business, Poznań, Poland.

Monika Guzek, Ph.D. candidate, Poznań University of Economics and Business, Poznań, Poland.

Correspondence concerning this article should be addressed to Małgorzata Bartosik-Purgat, Department of International Business, Poznań University of economics and Business, al. Niepodległości 10, 61-875 Poznań, Poland.
} 
Because of varying needs of users, a growing number of new media characterized by specific features have been appearing in the market, e.g. Instagram, Snapchat, Pinterest, Periscope, or Vine, where communication is mainly done through photos and short videos. For some people, mostly, the representatives of the $\mathrm{Z}$ generation, media such as Facebook or Twitter become unfashionable. They expect newer, more original, and more specialized solutions. Moreover, the results of the last research done by IRCenter show that more and more often users give up being active in several media simultaneously (Social Media 2014_-Polska Raport).

In the international market, a diversified way and use of particular SNS can be observed. It is caused by many factors among which cultural and political elements as well as the degree of development need to be distinguished. Many SNSs are available in the majority of countries in various parts of the world, however, in some markets, communities use local media which are often equivalents of the international ones (Ruleman, 2012). Such a situation takes place for example in China where the community mainly uses Youku instead of YouTube, while RenRen and Qzone are Chinese "substitutes" of Facebook and other Western media (Saw, Abbott, Donaghey, \& McDonald, 2013; Zhang \& Xueb, 2015).

The character and range of using social media are also influenced by different needs and personality traits (Khang, Han, \& Ki, 2014) of the residents of particular markets or regions. Due to these reasons, the factors which users take into account when choosing particular SNS are diversified. These factors are a special area of interest in this paper.

The main purpose of the paper is to identify the factors of SNS that influence individual users' choice regarding these media in foreign markets (however, it should be emphasized that the range of using media is not discussed by authors as one of the factors). Moreover, the detailed aims also include describing endogenous characteristics that differentiate this choice and indicating interdependence between the importance of particular factors of the media and the devices used by users. The literature studies presented in the first part of the article have been complemented with the empirical research done in the foreign markets. Due to a differentiated structure of the SNS market as well as the potential and directions of interests of companies, the authors focused in the article on three markets: Polish, American, and Chinese ones.

Knowledge about the factors that users take into account when choosing SNS, as well as indicating the significance of personality features that differentiate the choice, is important for both current and future creators of portals. Such information is also significant for companies to place the advertisements of their products there or using these media for another type of communication with potential buyers.

The structure of this article illustrates its main purpose. The first part includes an the literature review according to factors influencing the choice of social media and its determinants as age and gender. Then the methodology and the results of the empirical research are presented, next discussion and finally conclusions.

\section{Literature Review}

The literature broadly presents the results of projects related to the frequency and ways of using the Internet in general (Li \& Kirkupb, 2007; Lin \& Lu, 2011; Bolton, Parasuraman, Hoefnagels, Migchels, Kabadayi, Gruber, ... Solnet, 2013; Hamade, 2013), the area of using SNS (K. E. Howard, Curwen, N. R. Howard, \& Colon-Muniz, 2014; Hamid, Waycott, Kurnia, \& Chang, 2015; Sheldon, 2015), and the determinants differentiating this use. The attempts to indicate a correlation between ways of using SNS and personality of a user, e.g. how extraverts use social media and how it is done by introverts (Ross, E. S. Orr, 
Sisic, Arseneault, Simmering, \& R. R. Orr, 2009; Ryan \& Xenos, 2011; Nadkarni \& Hofmann, 2012) can most often be observed. In the literature, there are numerous publications presenting studies on using social media in the area of communication, entertainment, education, consumer behaviour, or work (Roblyer, McDaniel, Webb, Herman, \& Witty, 2010; Gammon \& White, 2011; Nicholas, Watkinson, Rowlands, \& Jubb, 2011; Ruleman, 2012; Tenopir, Volentine, \& King, 2013; Zhang, Liu, de Pablos, \& She, 2014), however, it is difficult to find the studies and scholarly publications which are closely related to the issues discussed in this article. The paper is an attempt to fill out a gap which exists in this area of the literature on the subject. This attempt aims at identifying the factors which are taken into account when choosing media by individuals (as well as the influence of the personal characteristics) in the international context. It needs to be emphasized that the authors of various scholarly publications refer to factors conditioning a choice of particular SNS, however, it is mainly done from the point of view of enterprises (Kaplan \& Haenlein, 2010; Ainin, Parveen, Moghavvemi, Jaafar, \& Shuib, 2015; Siamagka, Christodoulides, Michaelidou, \& Valvi, 2015), and not the point of view of an individual user, as it is presented in this article.

\section{Social Media in Foreign Markets}

One of the latest popularity rankings (according to the activity of users) of social networking services (SNS) across the world shows the predominance of Facebook (Figure 1), next places are held by Chinese Qzone, Google+, as well as Instagram and Twitter. Further places in the ranking are mainly related to the origin of social platforms, e.g. Baidu Tieba or Weibo; these media are used in the Chinese market (similarly to Qzone which is listed as the second one), while VKontakte is a popular social platform in Russia (Kemp, 2015; 2016).

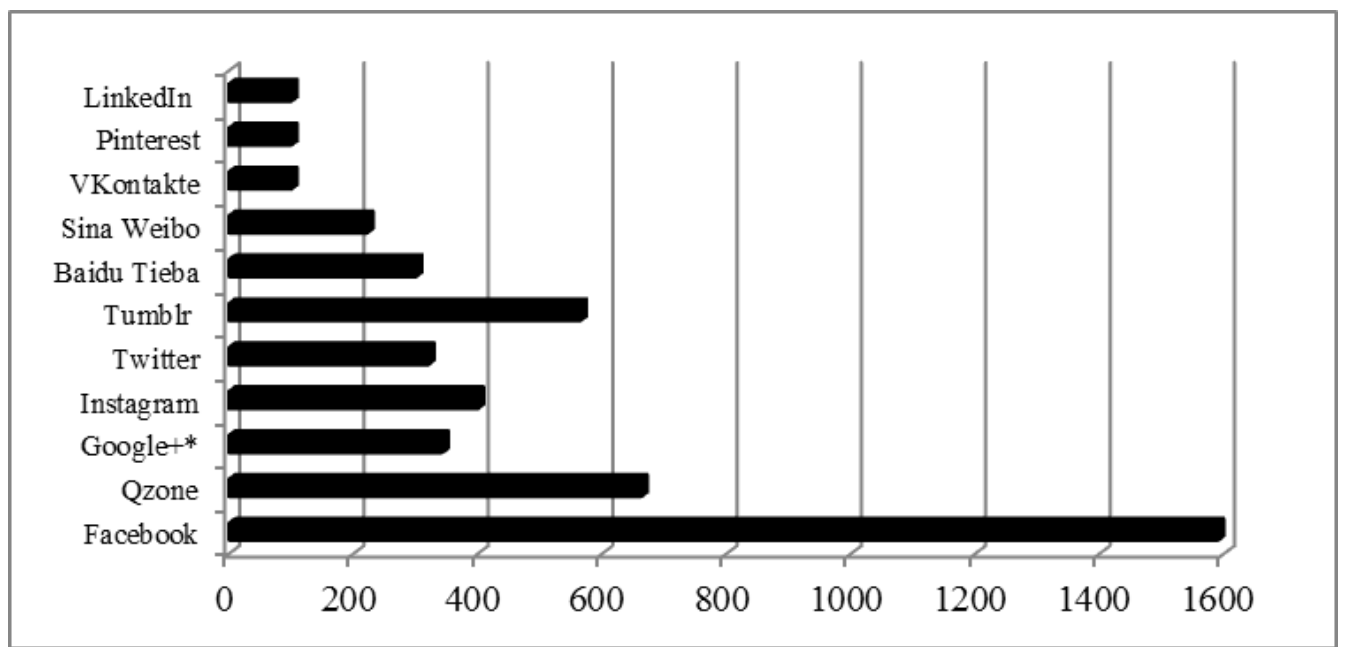

Figure 1. Number of active accounts - users of SNSs (in mln) in January 2016. Note. * data from 2015. Source: (Kemp, 2015; 2016).

The results of the research done by IRCenter in 2014 on the popularity and activity of Polish users in SNS indicate that the leading places, in terms of using a service, are continually held by YouTube (92\%) and Facebook (85\%), next by Twitter (28\%), and Instagram (25\%). These studies show that Facebook and Twitter kept the first two places (compared to the previous years) and that the activity of Polish users on internet forums decreased. There are also differences in the frequency of using between the genders. The vast majority of women prefer the blogosphere and Instagram. Men predominate in internet forums and as YouTube users. In 
Poland, both genders almost equally use Facebook (with a slight predominance of women 52\%/48\%), Twitter (women 55\%, men 45\%), or Google+ (56\%/44\%). The presented studies show that the activity of Polish women (in a form of publications) in SNS has been increasing in comparison to the years preceding the conducted research (by 7.3\%) (Social Media 2014—Polska Raport).

In the latest report on social media in the Chinese market, Kemp (2016) presented the most popular media divided into internet communicators and social services. It stems from these data that the dominant position among the communicators is held by WeChat (24\% of people indicated using this medium) which can be named an equivalent of Whatsapp, and Facebook Messenger with a significant difference in the number of users (only 4\%). Among SNS, the first place is occupied by Qzone (21\%) - the Chinese equivalent of Facebook, next by Sina Weibo (16\%), Baidu Tieba (14\%), Tencent Weibo (12\%), and Renren (6\%). Less popular are also the Chinese services such as Kaixin001 (5\%) and 51.com (3\%). In the discussed ranking, Facebook users were also observed, however, in comparison to the leader of the classification, their number is small-only $6 \%$ (Kemp, 2016).

The American market of SNS is dominated, in terms of the number of users, by one of the largest services, which is Facebook. Both the service as such, as well as the communicator, i.e. Facebook Messenger occupies the first places in the rankings. However, in 2015, a one-percent decrease in the number of Facebook users was observed, while the number of Facebook Messenger users increased by 6\% (Kemp, 2016). A typical user of Facebook in the United States is not necessarily a young person. A significant percentage of people in the age range of 55+ also use social websites here. The studies done by Experian Simmons show that in the group of people over 55 years old, $82 \%$ of Americans are social media users, while among people who turned 65 this percentage equals 73\% (Wilmowski, 2015). The measurement done by Realitymine in the American market shows the dominance of Facebook, regardless of age. The distinction is observed in relation to other services, e.g. Google+ is dominated by men from the Baby Boomer generation, the so-called image sharing services (Instagram, Pinterest) are definitely preferred by women from the Millennials generation (the number of women using Pinterest is five times higher than the number of men). Twitter is the only service in this research used mostly by American men, regardless of age (among its users the ones with higher education predominate) (Realitymine, 2015).

\section{Determinants of Choosing Social Networks}

Questions need to be asked here. What factors, besides the area of use, influence the choice of a particular SNS? Is it a number of people who already use it and access to a particular number of users? Is it the attractiveness of the interface, being user-friendly or maybe lack of a subscription fee?

In the literature, the factors of choosing SNS by individual users are very often associated with an area of use, e.g. communication and entertainment (Bohn, Buchta, Hornik, \& Mair, 2014), looking for study materials (Mora Mora, Signes Pont, Casado, \& Iglesias, 2015) and looking for a job (Zappa \& Robins, 2016; Chua, 2011). However, in this article, the authors focus on factors other than a way of using SNSs. Guerrero (2015), among the traits essential for the success of a SNS, listed the correct size of an image, its clarity as well as, at the same time, attractiveness and being striking, showing faces and smiles (people love to watch people), using slogans and keywords that attract the auditorium, recognisability through consistent use of the acknowledged signs and symbols and relevance of the placed information and images among others. The studies done among managers of small companies in the United States, the United Kingdom, Australia, and India showed that the factors 
influencing a choice of a particular social tool made by enterprises are the innovative character of media and their geographic range among others (Wamba \& Carter, 2013). Moreover, Zeiller and Schauer (2011) emphasized these social media characteristics which ensure providing important and high quality information and their significance for small enterprises. Other most often identified and mentioned determinants of choosing social media by enterprises are mainly compatibility (Y. M. Wang, Y. S. Wang, \& Yang, 2010; Ainin et al., 2015), costs related to using a particular medium, and its effectiveness (Chong \& Chan, 2012; Ainin et al., 2015), trust (Chai, Das, \& Rao, 2011), as well as interactivity and the possibility of reaching broad spectrum of users fast (Lee \& Kozar, 2012; Ainin et al., 2015).

Taking into account research achievements of other authors related to distinguishing the factors that influence a choice of SNSs made by individual users, the following research hypothesis has been formulated:

H1: A factor which to the greatest extent determines the choice of SNSs is its geographic range, and thereby the number of users.

The behaviour of SNS's users is influenced by a number of elements which differentiate the frequency of use, the range, and factors influencing the choice of particular services that are the subject of interest in this article. The determinants conditioning these behaviours are related to the individual characteristics of users, and in the article, they are described as endogenous factors. The studies done by other researchers in the area of using social media show that there is differentiation because of gender (Randall, Pauley, \& Culley, 2015; Ruleman, 2012; Muscanell \& Guadagno, 2012; McCormick \& Livett, 2012; Kozinets, De Valck, Wojnicki, \& Wilner, 2010) and age (Ruane \& Wallace, 2013; Lenhart, Purcell, Smith, \& Zickuhr, 2013; Brenner \& Smith, 2013). However, they mainly relate to the significances of using and not the factors of choosing services. The results of the studies where the variables differentiating the behaviour of social media users were endogenous factors, show interdependencies between them and ways of using these services. In other words, the factors such as gender and age differentiate the ways of using SNSs. On these grounds, the second hypothesis and two detailed hypotheses including endogenous characteristics of users of SNSs have been formulated.

$\mathrm{H} 2$ : Endogenous factors differentiate the significance of particular characteristics of social networking services.

H2a: Age of users differentiates the significance of the characteristics of social networking services when choosing them.

H2b: Gender of users differentiates the significance of the characteristics of social networking services when choosing them.

Another detailed aim described in the introduction of the article is to identify interdependencies between a type of a device applied to use social networking services and the importance of a characteristic feature of these services when choosing them by users. It is an interesting issue, because as a few years ago, the main device used for social networking was a desktop computer or laptop (West \& Thompson, 2015). Nowadays, technological development has affected a significant change in the accessibility of social media based on using mostly smartphones, tablets, or iPads for these purposes (Tan \& Goh, 2015). The number of people using SNS through mobile devices increases every year. For example, it stems from the report Digital, Social \& Mobile Worldwide in 2015 that in August 2015, 23\% increase in comparison to 2014 was noted (Kemp, 2015).

The results of the analyses done by Kemp in 2015 and 2016 show a great use of mobile devices, e.g. smartphones and tablets, for social purposes. For example, in Eastern Asia, over $89.6 \%$ of active social accounts are mobile accounts, in the North America, this rate is even higher and amounts to $93.8 \%$, in Western 
Europe 84\%, while in the South America as much as $74.8 \%$ (Kemp 2016). It also stems from the Digital in 2016 Report: We Are Social's, that the first place, with a significant advantage in the ranking, among the countries where the highest percentage of active social accounts in mobile devices in relation to the size of population was noted, was held by South Korea (76\%), next Qatar (66\%), Taiwan (64\%), United Aran Emirates (61\%), Iceland (60\%), Hong Kong ( 59\%), Singapore (58\%), as well as Malesia and the United States (52\% each).

The increasing number of people using mobile devices prompts to ponder what is the evaluation of the importance of the characteristics of SNSs when making a choice. To this end, the third hypothesis indicating the interdependence between a type of a device and the evaluation of the characteristics of SNSs pointed out by users as important when choosing a medium has been formulated.

H3: The evaluation of the importance of the characteristics of social networking services is influenced by the type of devices applied by users.

\section{Research Methods}

Empirical research was conducted with the use of a quantitative research method such as paper and electronic inquiry forms (CAWI), filled out by the respondents themselves. The standardized questionnaire is an original instrument prepared for the purpose of the described research. The only element differentiating the measurement instrument in the researched markets was the language. In order to gather a greater number of filled-out questionnaires, the Polish language was applied in Poland, Chinese in China, and English in the United States. The original language of the questionnaire was Polish, next it was translated into English and Chinese by means of forward and backward translation. It was based on translating the questionnaire from Polish into particular languages, and next again into Polish. The original version was compared with the final one in order to eliminate errors stemming from linguistic, lexical, or context differences (Adams, Madhavan, \& Simon, 2006).

\section{Sampling Method and Sample Size}

Collecting the data in the empirical research was finished at the end of 2015 and the beginning of 2016. In the three countries analysed in this article, the total number of the respondents participating in the research was 851, including 295 from China, 296 from Poland, and 260 from the United States. The respondents were selected by means of one of nonprobability sampling methods (snow ball sampling). In each market, there were people responsible for providing the respondents with questionnaires, either in a paper or electronic version. Applying the online questionnaire did not result in significant response rate $(3.5 \%$ of all the collected questionnaires). The applied method of sampling produces the consequences related to the interpretation of the obtained results which, in the discussed case, should not be fully generalized for the population of the researched countries, SNS's users. They are characteristic of particular groups participating in the survey.

\section{Sample Profile}

The researched groups in particular countries are differentiated by endogenous criteria, among which gender and age were analysed. Table 1 presents the detailed data obtained in particular countries. It needs to be emphasized that in terms of gender, similar distribution was obtained in the Chinese and Polish groups, where the majority (around $70 \%$ of the respondents) were women. In the United States, the group was almost balanced in terms of the participation of men and women. 
Table 1

Respondents' Profile in Terms of the Chosen Endogenous Criteria (\%)

\begin{tabular}{lllll}
\hline Endogenous features of respondents & Categories & China & Poland & United States \\
\hline Gender & & & & \\
\hline & Women & 68.1 & 70.9 & 56.5 \\
& Men & 31.2 & 27.7 & 43.1 \\
& No data & 0.7 & 1.3 & 0.4 \\
\hline Age & & & & \\
\hline & 15-20 years old & 14.2 & 35.5 & 79.6 \\
& 21-30 years old & 66.4 & 63.5 & 16.9 \\
& 31 years old and older & 19 & 0.7 & 3.5 \\
\hline
\end{tabular}

Source: own research.

In terms of age, in the Polish and Chinese groups, the largest parts were people aged between 21-30. In the United States, the age group of 15-20 predominated. In the American and Polish groups, the fewest respondents were from the 31 and older groups, while in China, this class included $19 \%$ of the respondents.

\section{Research Results}

In order to describe the most important factors (F) of SNSs which are taken into account by users choosing a service, the respondents were asked to point out the five most significant ones from the prepared list of categories. In order to examine the interdependencies between the endogenous characteristics of users (gender and age) and the factors of SNSs, the analysis of statistical indexes demonstrating an interdependence/or its lack was done. In order to determine the importance of the occurrence of differences between the endogenous factors of users and the characteristics of SNSs (generally, without making a distinction between particular services), Pearson's chi-square statistics was applied. In order to establish the strength of the correlation, Cramer's $V$ factor was used. Applying the distinguished statistics stems from the characteristics of the scales of measurement used in the questionnaire. Similarly, in order to determine the interdependence between the indicated factors of SNSs and a type of used devices employed by users, Pearson's chi-square statistics was applied.

\section{Factors of SNSs}

In all the researched countries (Figure 2), firstly, the number of people (F3), which a user has access to through a particular medium; secondly, lack of fees for using a service (F7); and, thirdly, being user-friendly was indicated as the most significant characteristic of SNSs (F2). Large convergence was observed in the three researched countries in terms of these three characteristics. The least important for the respondents were the name of the service (F9), availability of social games (F5), as well as presence of advertisements of various products (F11). The greatest differences among the researched countries were obtained in the case of the availability of social games (in China, $4.4 \%$ of the respondents indicated this characteristic as important, while in Poland and the United States around 18\% in each of the countries), ease of activating an account (the largest number of Poles indicated this feature- $70.2 \%$ and the fewest were Americans-51.1\%), great diversity of functions (62\% of the Chinese respondents emphasized the importance of this feature when choosing SNSs, $43.6 \%$ of Poles, and $34.6 \%$ of Americans), and the possibility of using a mobile application of a service (38.9\% of the Chinese respondents, $49.6 \%$ of the Polish respondents, and $61.1 \%$ of Americans). 


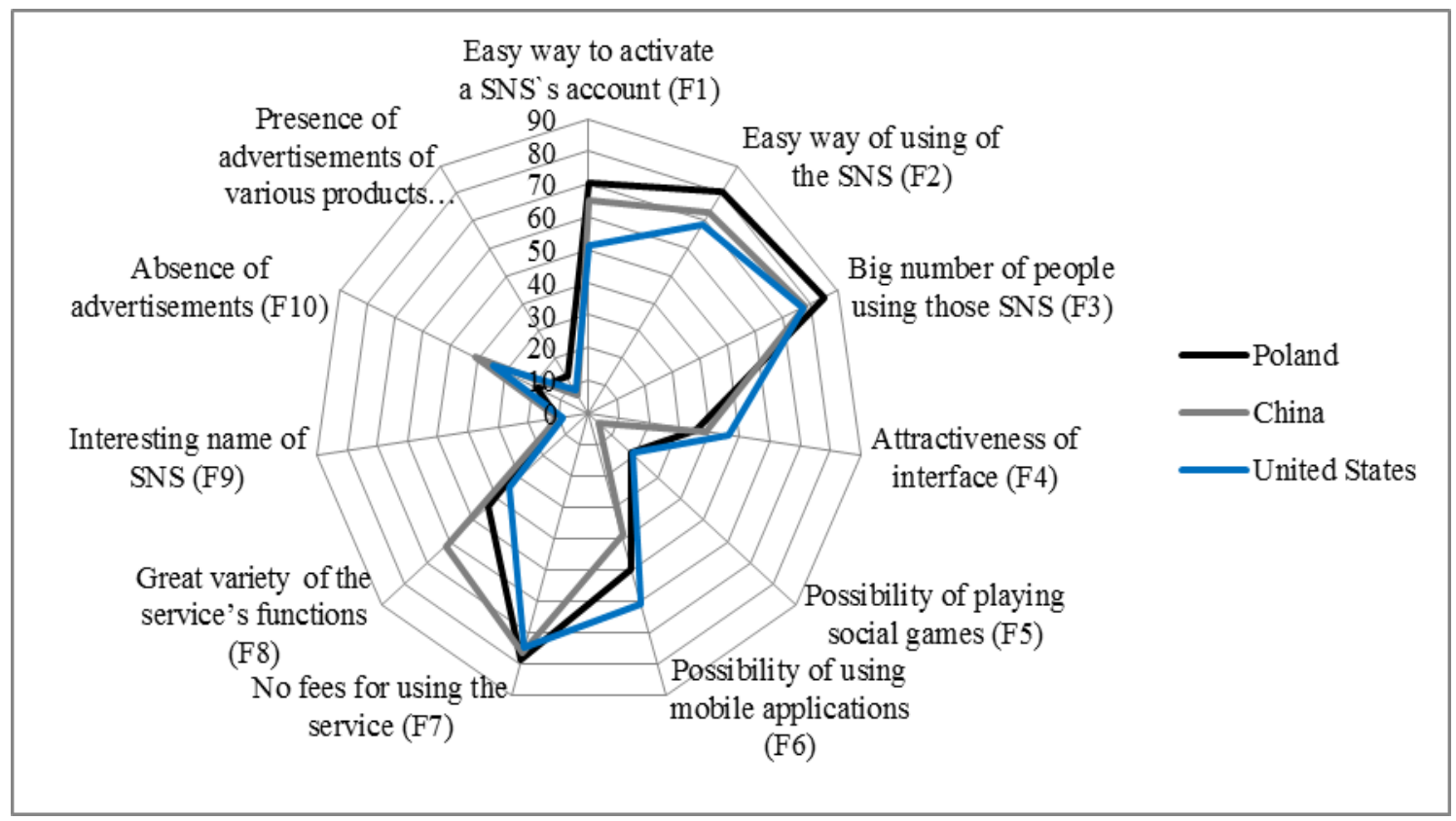

Figure 2. Factors of SNSs taken into account when choosing (\% of indications). Source: own research.

Among the characteristics that influence the choice of a particular service, in the Chinese group, the majority of the respondents indicated the number of people using a given medium (77.9\%), lack of fees for using a service $(76.6 \%)$, ease of use $(73.2 \%)$, registering an account $(65.1 \%)$, and variety of functions $(62 \%)$. Possibility of playing games through a given service turned out to be the least important for the Chinese respondents (4.4\%). Similarly, in the Polish group, the factors of the SNSs used by the respondents (which are, at the same time, elements influencing the choice) are mainly: large community (84.4\%), being user-friendly and easy to use $(80.4 \%)$, as well as lack of fees for using a service $(78.8 \%)$. The name of a service $(10.1 \%)$, advertisements placed in a service (13.2\%), and possibility of playing games through these services (18.2\%) are the least important for the Polish respondents. As far as the respondents from the American group are concerned the characteristics they pay attention to when choosing a service, which are also: great number of people - users $(77.3 \%)$, lack of fees for using $(74.6 \%)$, ease of use $(68.8 \%)$, and the possibility of using a service via a mobile device $(61.1 \%$ ) - this is the highest result among the researched countries related to the possibility of using SNSs through mobile devices. The name of a service $(8.8 \%)$ or advertisements placed there $(8.1 \%)$ are almost of no importance here.

\section{Properties of SNSs and the Endogenous Characteristics of Users}

The statistical analysis with the use of chi-square test showed a correlation between the gender of the respondents and the characteristic factors of SNSs described as a possibility of using a mobile application of a service (F6) in all the presented countries (Table 2). In other words, gender differentiates the significance of this factor when choosing SNSs. In China, women pay more attention to accessing services through mobile devices $(p<0.01 ; V=0.14)$, however, the strength of the relation between gender and this feature is established here, on the basis of $V$ Cramer, as weak. Similarly, in the two other countries, women to a greater extent take into account F6 when choosing SNSs, however, the strength of these relations is also at the weak level ( $p<$ $0.05 ; V=0.12)$. In the United States, chi-square test indicated the significance of the interdependence between 
gender and F1. American women more often pay attention to whether it is easy to activate an account in SNSs $(p<0.05 ; V=0.12)$. In the case of other characteristics, gender does not differentiate the significance of particular factors of SNSs.

Table 2

Correlations Between the Factors of SNSs and Gender

\begin{tabular}{lllllll}
\hline \multirow{2}{*}{ Symbol } & \multicolumn{2}{c}{ China } & \multicolumn{2}{c}{ United States } \\
\cline { 2 - 7 } & $x^{2}$ & $V$ & $x^{2}$ & $V$ & $x^{2}$ & 0.12 \\
\hline F1 & 1.96 & 0.08 & 0.2 & 0.01 & $4.1^{* *}$ & 0.02 \\
F2 & 0.38 & 0.03 & 0.97 & 0.05 & 0.14 & 0.009 \\
F3 & 0.4 & 0.03 & 1.4 & 0.07 & 0.02 & 0.08 \\
F4 & 0.15 & 0.02 & 0.003 & 0.003 & 1.65 & 0.06 \\
F5 & 0.6 & 0.04 & 1.65 & 0.07 & 1.09 & 0.12 \\
F6 & $6.01^{*}$ & 0.14 & $4.34^{* *}$ & 0.12 & $3.99^{* *}$ & 0.037 \\
F7 & 0.01 & 0.006 & 0.6 & 0.04 & 0.37 & 0.015 \\
F8 & 0.14 & 0.02 & 0.49 & 0.04 & 0.06 & 0.05 \\
F9 & 1.01 & 0.06 & 1.22 & 0.06 & 0.8 & 0.008 \\
F10 & 1.25 & 0.06 & 0.03 & 0.01 & 0.01 & 0.039 \\
F11 & 0.003 & 0.001 & 0.8 & 0.05 & 0.4 &
\end{tabular}

Notes. * Significance level $0.01 ; * *$ significance level 0.05 .

In relation to age, as an endogenous variable conditioning the choice of SNSs in terms of their specific factors, it can be underlined that age is a variable differentiating the significance of those factors to the greatest extent in the Chinese group. The influence of this variable on the three factors was observed here. In the case of F5 and F9, the youngest Chinese respondents (15-20) particularly take these factors into account $(p<0.05 ; V=$ 0.15), when choosing SNSs. Lack of advertisements in a given service (F10) is important for slightly older Chinese respondents (21-30) $(p<0.05 ; V=0.15)$. In the Polish group, age significantly influences the importance of a characteristic related to the ease of using a service (F2). This characteristic turned out to be particularly important for the Polish respondents from the 21-30 age group ( $p<0.01 ; V=0.18$ ). As far as the youngest Polish respondents (15-20) are concerned the most important factors turned out to be the limited number of advertisements in services (F10) $(p<0.01 ; V=0.17)$. For the youngest American respondents, the fact that using a particular service makes it possible to reach a large community of users (F3) $(p<0.01 ; V=$ 0.22 ) is important. In relation to the remaining characteristics, the respondents (regardless of age) behave similarly in terms of evaluating the distinguished variables when choosing SNSs.

\section{Properties of SNAs and a Type of Mobile Devices}

Referring to a type of devices used by the respondents when using SNS, three devices most often indicated in particular groups are needed to be distinguished. These devices are included in the category of the so-called portable or mobile devices, i.e. laptop, tablet/iPad, or smartphone. Only some respondents from the studied groups use desktop computers, however, they were not analysed.

Laptop. In the studied groups (Chinese and American), the same relations were noted. The first one indicates the interdependence between a factor of SNSs which is the attractiveness of interface (F4) and employing laptops to use these media. In other words, using a laptop significantly influences the importance of the F4 characteristic in the Chinese $(p<0.01 ; V=0.24)$ and American $(p<0.05 ; V=0.18)$ groups. Furthermore, 
in these two groups, using a laptop in order to access SNSs significantly influences the importance of a factor which is related with great variety of their functions (F8) $(p<0.02 ; V=0.18)$. In the Polish group, using a laptop significantly influences the importance of F11 which is the presence of advertisements. In other words, the researched poles using SNSs through laptops pay attention to the presence of advertisements of different products in these services $(p<0.05 ; V=0.18)$.

Table 3

Correlations Between the Factors of SNSs and Age

\begin{tabular}{lllllll}
\hline \multirow{2}{*}{ Symbol } & \multicolumn{2}{c}{ China } & \multicolumn{2}{c}{ Uoland } & \multicolumn{2}{c}{ United States } \\
\cline { 2 - 7 } & $x^{2}$ & $V$ & $x^{2}$ & $V$ & 3.6 & 0.11 \\
F1 & 0.84 & 0.05 & 1.41 & 0.07 & 0.03 & 0.01 \\
F2 & 0.004 & 0.003 & $9.7^{*}$ & 0.18 & $12.4^{*}$ & 0.22 \\
F3 & 0.24 & 0.03 & 3.5 & 0.12 & 2.24 & 0.09 \\
F4 & 1.95 & 0.08 & 1.76 & 0.07 & 3.8 & 0.12 \\
F5 & $6.8^{* *}$ & 0.15 & 3.8 & 0.11 & 3.58 & 0.11 \\
F6 & 2.6 & 0.09 & 0,32 & 0.03 & 0.46 & 0.04 \\
F7 & 0.24 & 0.03 & 5.6 & 0.13 & 0.07 & 0.01 \\
F8 & 0.27 & 0.03 & 0.05 & 0.013 & 0.31 & 0.03 \\
F9 & $6.3^{* *}$ & 0.15 & 0.48 & 0.04 & 0.92 & 0.06 \\
F10 & $6.4^{* *}$ & 0.15 & $9.1^{*}$ & 0.17 & 3.48 & 0.11 \\
F11 & 2.86 & 0.09 & 0.44 & 0.04 &
\end{tabular}

Notes. * Significance level $0.01 ; * *$ significance level 0.05 .

Table 4

Correlations Between the Importance of SNS's Factors and Using Mobile Devices-Laptop

\begin{tabular}{|c|c|c|c|c|c|c|}
\hline \multirow{2}{*}{ Symbol } & \multicolumn{2}{|l|}{ China } & \multicolumn{2}{|c|}{ Poland } & \multicolumn{2}{|c|}{ United States } \\
\hline & $\overline{x^{2}}$ & $V$ & $x^{2}$ & $V$ & $x^{2}$ & $V$ \\
\hline $\mathrm{F} 1$ & 7.9 & 0.17 & 0.6 & 0.04 & 4.7 & 0.13 \\
\hline $\mathrm{F} 2$ & 3.3 & 0.1 & 4.77 & 0.12 & 0.6 & 0.05 \\
\hline F3 & 7.7 & 0.16 & 5.58 & 0.14 & 6.4 & 0.15 \\
\hline $\mathrm{F} 4$ & $15.9^{*}$ & 0.24 & 3.3 & 0.1 & $8.5^{* *}$ & 0.18 \\
\hline F5 & 3.76 & 0.11 & 7.4 & 0.16 & 2.9 & 0.1 \\
\hline F6 & 5.5 & 0.14 & 1.46 & 0.07 & 2.15 & 0.09 \\
\hline F7 & 1.9 & 0.08 & 1.8 & 0.08 & 0.4 & 0.04 \\
\hline F8 & $8.8^{* *}$ & 0.18 & 6.1 & 0.14 & $8.2^{* *}$ & 0.18 \\
\hline F9 & 3.6 & 0.11 & 5.5 & 0.13 & 0.07 & 0.02 \\
\hline F10 & 1.3 & 0.06 & 2.6 & 0.09 & 0.42 & 0.04 \\
\hline F11 & 6.5 & 0.15 & $9.2^{* *}$ & 0.18 & 2.4 & 0.09 \\
\hline
\end{tabular}

Notes. * Significance level $0.01 ; * *$ significance level 0.05 .

Tablet/iPad. In the case of using SNSs through tablets, more varied interdependencies were observed than in the group of the respondents using laptops. Chi-square test showed in the Chinese group the relation between these users and the importance of F6, i.e. possibility of using a mobile application of a service $(p<0.05 ; V=$ $0.18)$.

In the Polish group, the influence of tablet on F2 $(p<0.05 ; V=0.18)$ and F3 $(p<0.05 ; V=0.2)$ was noted. In the American group tablet users, more than other ones, value higher lack of fees for using social networking services (F7) $(p<0.05 ; V=0.2)$. 
Table 5

Correlations Between the Importance of SNS's Factors and Using Mobile Devices-Tablet/iPad

\begin{tabular}{lllllll}
\hline Symbol & \multicolumn{3}{c}{ China } & \multicolumn{3}{c}{ United States } \\
\cline { 2 - 7 } & $x^{2}$ & $V$ & $x^{2}$ & $V$ & $x^{2}$ & \multicolumn{2}{c}{ Poland } \\
\hline F1 & 7.09 & 0.16 & 4.7 & 0.12 & 1.8 & 0.08 \\
F2 & 1.3 & 0.07 & $9.16^{* *}$ & 0.18 & 0.2 & 0.03 \\
F3 & 3.3 & 0.1 & $11.5^{* *}$ & 0.2 & 2.7 & 0.1 \\
F4 & 5.6 & 0.14 & 0.6 & 0.04 & 4.2 & 0.13 \\
F5 & 4.4 & 0.12 & 5.9 & 0.14 & 3.8 & 0.12 \\
F6 & $8.7^{* *}$ & 0.18 & 5.99 & 0.14 & 7.08 & 0.16 \\
F7 & 2.9 & 0.1 & 0.5 & 0.04 & $10.4^{* *}$ & 0.2 \\
F8 & 3.0 & 0.1 & 2.2 & 0.08 & 1.8 & 0.08 \\
F9 & 0.6 & 0.04 & 3.2 & 0.1 & 6.7 & 0.16 \\
F10 & 1.7 & 0.07 & 4.8 & 0.13 & 2.8 & 0.1 \\
F11 & 4.8 & 0.13 & 8.2 & 0.17 & 4.7 & 0.13 \\
\hline
\end{tabular}

Note. $* *$ significance level 0.05 .

Smartphones. The greatest number of interdependencies between a type of a device and the importance of the factors of SNSs was observed in the group of smartphone users. In all the researched countries, using a smartphone significantly influences the importance of F6 which is a possibility of using a mobile application (China: $p<0.01 ; V=0.2$; Poland: $p<0.05 ; V=0.17$; the United States: $p<0.05 ; V=0.22$ ). This interdependence is related to the degree of mobility as smartphones are considered to be the most mobile among mobile devices. Moreover, in the Chinese and American groups, significant influence of the nature of a device - smartphone - on the importance of F3, i.e. a big number of people using services was presented. The strength of this relation in the Chinese group was established at the moderate level $V=0.3(p<0.01)$, in the American group, this degree is weak $V=0.2(p<0.01)$.

Table 6

Correlations Between the Importance of SNS's Factors and Using Mobile Devices-Smartphone/iPhone

\begin{tabular}{lllllll}
\hline Symbol & China & \multicolumn{3}{c}{ United States } \\
& $x^{2}$ & $V$ & $x^{2}$ & $V$ & $x^{2}$ & $V$ \\
\hline F1 & 6.6 & 0.15 & 5.7 & 0.14 & 2.3 & 0.09 \\
F2 & $11.3^{* *}$ & 0.19 & 2.5 & 0.09 & 2.5 & 0.09 \\
F3 & $25.6^{*}$ & 0.3 & 4.65 & 0.12 & $10.3^{*}$ & 0.2 \\
F4 & 1.6 & 0.07 & 2.65 & 0.09 & 7.01 & 0.16 \\
FS5 & $10.4^{*}$ & 0.19 & 6.06 & 0.14 & 1.09 & 0.06 \\
F6 & $11.9^{*}$ & 0.2 & $8.5^{* *}$ & 0.17 & $12.3^{*}$ & 0.22 \\
F7 & 8.1 & 0.17 & 6.7 & 0.15 & 7.2 & 0.16 \\
F8 & $18.6^{*}$ & 0.25 & 1.8 & 0.08 & 8.2 & 0.17 \\
F9 & 1.1 & 0.06 & 1.6 & 0.07 & 1.5 & 0.07 \\
F10 & 4.9 & 0.13 & 3.17 & 0.1 & 1.8 & 0.08 \\
F11 & 1.12 & 0.06 & $8.85^{* *}$ & 0.17 & 1.7 & 0.08 \\
\hline
\end{tabular}

Notes. * Significance level $0.01 ; * *$ significance level 0.05 .

Among the researched Chinese respondents, a significant correlation between the importance of the F2 ( $p$ $<0.05 ; V=0.19), \mathrm{F} 5(p<0.01 ; V=0.19)$, and F8 $(p<0.01 ; V=0.25)$ characteristics was also observed. In the 
Polish group, the influence of employing smartphones in order to use SNSs on the presence of advertisements of various products $(\mathrm{F} 11)(p<0.05 ; V=0.17)$ was obtained.

\section{Discussion}

The analysis of the factors which are significant when choosing SNSs showed that all the respondents (regardless of a country) considered the following aspects as the most important ones: firstly, the number of people (F3) a user has access to through a given service; secondly, lack of fees for using them (F7); and thirdly, ease of use (F2). The presented findings are also convergent with the results obtained in other research projects (Wamba \& Carter, 2013). Therefore, the first hypothesis which was formulated after the literature studies and which states that a factor which to the greatest extent determining the use of social networking services is its geographic range, and thereby the number of users, has been validated.

Statistical calculations done in a form of chi-square test demonstrated that in all the researched countries there is a correlation between the gender of the respondents and the factors related to the possibility of using a mobile application of a service (F6). Women value higher F6 than men when deciding about the choice of SNS. It can be therefore stated that $\mathrm{H} 2 \mathrm{a}$ is true in relation to this factor of SNSs. Taking into account the results differentiating the evaluation of the importance of the factors of SNS while choosing them, it should be stated that age is a significant variable especially in the Chinese group. In each of the remaining groups, one correlation between age and the factors of SNSs was obtained. It can be therefore concluded that $\mathrm{H} 2 \mathrm{~b}$ is true. The analysis of these interdependencies shows that in the Chinese group, the youngest respondents pay the greatest attention to the possibility of playing social games and an interesting name of a social service. Older Chinese respondents take into account lack of advertisements on the SNSs they use. An interesting fact was noted in the Polish group, where the younger respondents considered the factor related to lack of advertisements as important, while the older ones paid more attention to the ease of use.

The above results have an application value as the knowledge derived from them can be very useful to the creators of SNSs. The information about the importance of characteristics for particular groups of users will make it possible to adjust the offer to their expectations, e.g. services directed toward younger users to a greater extent should enable them to play games through these websites. The youngest Chinese users, however, pay also attention to the name of a service, which is equally important from the point of view of their creators. Services whose users are supposed to be mainly women should offer mobile applications.

The results of the presented studies and carried out analyses also make it possible to determine the attitude of particular users toward advertisements that appear in social media. The characteristic of a service related to the presence or lack of advertisements in it was particularly correlated with age in the Chinese and Polish groups. What is interesting, the Chinese from the age group of 21-30 prefer the services where the presence of advertisements is limited to minimum. Another interesting fact was observed in the Polish group where lack of advertisements was indicated as an important factor influencing the choice of a service in the group of the youngest respondents (age range of 15-20). This information is important for both the creators of SNSs directed towards the segment of younger users, as well as advertisers. The surplus of advertisements on a website, if they are not welcomed, might have negative influence on the attitude towards a service as well as advertised products.

Referring to mobile devices whose users evaluate the importance of particular characteristics of SNSs, it should be highlighted that the largest number of interdependencies were noted among the respondents using 
smartphones. Smartphones users, regardless of a country, pay particular attention to the possibility of using SNSs through mobile applications. What is interesting, users of laptops, from two countries - China and Poland-pay attention to the attractiveness of interface of SNSs. It might be related to the limited size of screens in smartphones and tablets. The observed interdependencies between using mobile devices and the evaluation of the importance of particular factors of SNSs allow determining that the hypothesis $\mathrm{H} 3$ stating that the evaluation of the importance of the characteristics of SNSs is influenced by the type of devices applied by users is true.

\section{Conclusions}

Summing up the conclusions which stem from the literature studies and the statistical analysis of the obtained results in the three culturally diversified groups, it needs to be stated that there are slight differences between the countries in terms of the importance of the factors of SNSs. Endogenous characteristics of users, such as gender and age, differentiate the importance of particular variables. In some cases (e.g. gender), these interdependencies are similar in all three researched groups. However, as far as most aspects are concerned, the results vary. The greatest interdependencies between endogenous characteristics of users and the elements of SNSs were noted in the Chinese group. The creators of services should take into account these characteristics particularly in this market when preparing new offers and implementing changes in already existing ones.

The research also indicated great significance of mobile devices in using SNSs. Slightly different factors are important for laptop users than for smartphone users. The presented results have a significant application value for business entities creating SNS and their mobile applications in the foreign market. These results indicate common areas, as well as the ones that should be adjusted to the expectations of particular users.

\section{References}

Adams, A. M., Madhavan, S., \& Simon, D. (2006). Measuring social networks cross-culturally. Social Networks, 28(4), 363-376. Ainin, S., Parveen, F., Moghavvemi, S., Jaafar, N. I., \& Shuib, N. L. M. (2015). Factors influencing the use of social media by SMEs and its performance outcomes. Industrial Management \& Data Systems, 115(3), 570-588.

Bohn, A., Buchta, C., Hornik, K., \& Mair, P. (2014). Making friends and communicating on Facebook: Implications for the access to social capital. Social Networks, 37, 29-41.

Bolton, R. N., Parasuraman, A., Hoefnagels, A., Migchels, N., Kabadayi, S., Gruber, T., ... Solnet, D. (2013). Understanding Generation Y and their use of social media: A review and research agenda. Journal of Service Management, 24(3), $245-267$.

Brenner, J., \& Smith, A. (2013). 72\% of online adults are social networking site users. Pew Research Center. Retrieved from http://www.pewinternet.org/ /media/Files/Reports/2013/PIP_Social_networking_sites_update.pdf

Chai, S., Das, S., \& Rao, H. R. (2011). Factors affecting bloggers' knowledge sharing: An investigation across gender. Journal of Management Information Systems, 28(3), 309-334.

Chong, A. Y. L., \& Chan, F. T. (2012). Structural equation modelling for multi-stage analysis on radio frequency identification (RFID) diffusion in the health care industry. Expert Systems With Applications, 39(10), 8645-8654.

Chua, V. (2011). Social networks and labour market outcomes in a meritocracy. Social Networks, 33(1), 1-11.

Gammon, M. A., \& White, J. (2011). (Social) media literacy: Challenges and opportunities for higher education. Educating Educators with Social Media, 1, 329-345.

Guerrero, A. (2015). Eight traits that all successful social media images must have. Retrieved from http://www.postplanner.com/traits-of-successful-social-media-images/

Hamade, S. N. (2013). Perception and use of social networking sites among university students. Library Review, 62(6/7), 388-397.

Hamid, S., Waycott, J., Kurnia, S., \& Chang, S. (2015). Understanding students' perceptions of the benefits of online social networking use for teaching and learning. The Internet and Higher Education, 26, 1-9.

Howard, K. E., Curwen, M. S., Howard, N. R., \& Colon-Muniz, A. (2014). Attitudes toward using social networking sites in educational settings with underperforming latino youth: A mixed methods study. Urban Education, 1-30. 
Kaplan, A. M., \& Haenlein, M. (2010). Users of the world, unite! The challenges and opportunities of social media. Business Horizons, 53(1), 59-68.

Kemp, S. (2015). Digital, Social \& Mobile Worldwide in 2015. Retrieved from http://wearesocial.net/blog/2015/01/digital-social-mobile-worldwide-2015.

Kemp, S. (2016). Digital in 2016 report: We are social's. Retrieved from http://wearesocial.com/uk/special-reports/digital-in-2016.

Khang, H., Han, E.-K., \& Ki, E.-J. (2014). Exploring influential social cognitive determinants of social media use. Computers in Human Behavior, 36, 48-55.

Kozinets, R. V., de Valck, K., Wojnicki, A. C., \& Wilner, S. J. S. (2010). Networked narratives: Understanding word-of-mouth marketing in online communities. Journal of Marketing, 74(2), 71-89.

Lee, Y., \& Kozar, K. (2012). Developing a theory of website usability: An exploratory study to identify constructs and nomological networks. Decision Support Systems, 52(2), 450-463.

Lenhart, A., Purcell, K., Smith, A., \& Zickuhr, K. (2013.) Social media and mobile internet use among teens and young adults. Pew Internet \& American Life Project (February).

Li, N., \& Kirkupb, G. (2007). Gender and cultural differences in Internet use: A study of China and the UK. Computers \& Education, 48(2), 301-317.

Lin, K. Y., \& Lu, H. P. (2011). Why people use social networking sites: An empirical study integrating network externalities and motivation theory. Computers in Human Behavior, 27(3), 1152-1161.

McCormick, H., \& Livett, C. (2012). Analysing the influence of the presentation of fashion garments on young consumers' online behaviour. Journal of Fashion Marketing and Management, 16(1), 21-41.

Mora Mora, H., Signes Pont, M. T., Casado, G., \& Iglesias, V. G. (2015). Management of social networks in the educational process. Computers in Human Behavior, 51, 890-895.

Muscanell, N. L., \& Guadagno, R. E. (2012). Make new friends or keep the old: Gender and personality differences in social networking use. Computers in Human Behavior, 28, 107-112.

Nadkarni, A., \& Hofmann, S. G. (2012). Why do people use Facebook? Personality and Individual Differences, 52(3), $243-249$.

Nicholas, D., Watkinson, A., Rowlands, I., \& Jubb, M. (2011). Social media, academic research and the role of university libraries. Journal of Academic Librarianship, 37(5), 373-375.

Randall, N. H., Pauley, S. C., \& Culley, A. B. (2015). Family social networks, reciprocal socialization and the adoption of social media by baby boomer and silent generation women. Studies in Media and Communication, 9, 135-160.

Realitymine. (2015). Demographics of Social Media Users by Channel. Retrieved from http://www.realitymine.com/demographics-of-social-media-users/

Roblyer, M. D., McDaniel, M., Webb, M., Herman, J., \& Witty, J. V. (2010). Findings on Facebook in higher education: A comparison of college faculty and student uses and perceptions of social networking sites. Internet and Higher Education, 13, 134-140.

Ross, C., Orr, E. S., Sisic, M., Arseneault, J. M., Simmering, M. G., \& Orr, R. R. (2009). Personality and motivations associated with Facebook use. Computers in Human Behavior, 25(2), 578-586.

Ruane, L., \& Wallace, E. (2013). Generation Y females online: Insights from brand narratives. Qualitative Market Research. An International Journal, 16(3), 315-335.

Ruleman, A. B. (2012). Social media at the university: A demographic comparison. New Library World, 113(7/8), 316-332.

Ryan, T., \& Xenos, S. (2011). Who uses Facebook? An investigation into the relationship between the Big Five, shyness, narcissism, loneliness, and Facebook usage. Computers in Human Behavior, 27(5), 1658-1664.

Saw, G., Abbott, W., Donaghey, J., \& McDonald, C. (2013). Social media for international students-It's not all about Facebook. Library Management, 34(3), 156-174.

Sheldon, P. (2015). Understanding students' reasons and gender differences in adding faculty as Facebook friends. Computers in Human Behavior, 53, 58-62.

Siamagka, N. T., Christodoulides, G., Michaelidou, N., \& Valvi, A. (2015). Determinants of social media adoption by B2B organizations. Industrial Marketing Management, 51, 89-99.

Social Media 2014 - Polska Raport. (2014). Retrieved from http://ircenter.com/social-media-2014-polska/

Tan, E. M. Y., \& Goh, D. H. L. (2015). Presenting social media information on mobile devices using multiple contexts. Aslib Journal of Information Management, 67(2), 182-202.

Tenopir, C., Volentine, R., \& King, D. W. (2013). Social media and scholarly reading. Online Information Review, 37(2), $193-216$. 
Wamba, S. F., \& Carter, L. (2013). Twitter adoption and use by SMEs: An empirical study in System Sciences (HISS). 46th Hawaii International Conference on System Sciences, 2052-2049.

Wang, Y. M., Wang, Y. S., \& Yang, Y. F. (2010). Understanding the determinants of RFID adoption in the manufacturing industry. Technological Forecasting and Social Change, 77(5), 803-815.

West, D., \& Thompson, S. (2015). Mobile knowledge: Driving a paradigm shift. Journal of Applied Research in Higher Education, 7(1), 43-54.

Wilmowski, M. (2015). Ponad 90\% internautów w USA używa serwisów społecznościowych. Retrived from http://gadzetomania.pl/9938,ponad-90-internautow-w-usa-uzywa-serwisow-spolecznosciowych

Zappa, P., \& Robins, G. (2016.) Organizational learning across multi-level networks. Social Networks, 44, 295-306.

Zeiller, M., \& Schauer, B. (2011). Adoption, motivation and success factors of social media for team collaboration in SMEs. Proceeding of The 11th International Conference on Knowledge Management and Knowledge Technologies, Graz.

Zhang, X., Liu, L., de Pablos, P. O., \& She, J. (2014). The auxiliary role of information technology in teaching: Enhancing programming course using alice. International Journal of Engineering Education, 30(3), 560-565.

Zhang, Z., \& Xueb, Y. (2015). An investigation of how Chinese university students use social software for learning purposes. Procedia-Social and Behavioral Sciences, 186, 70-78. 